Complementary

Medicine Research
Abstract Service

Complement Med Res 2020;27:123-125

DOI: 10.1159/000506820

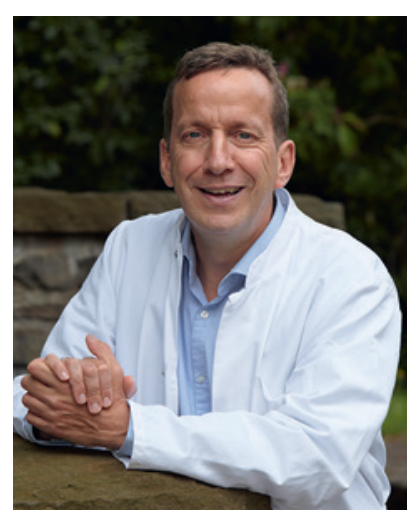

\section{Artikel, die Sie nicht versäumen sollten}

\author{
Ausgewählt durch Jost Langhorst, Bamberg
}

\section{Piovani D, Danese S, Peyrin-Biroulet L, Nikolopoulos GK, Lytras T, Bonovas S. Environmental Risk Factors for Inflammatory Bowel Diseases: An Umbrella Review of Meta-Analyses. Gastroenterology. 2019 Sep;157(3):647-659.}

Der „Umbrella-Review“ stellt die Zusammenfassung von Metaanalysen zu Umwelt-faktoren als Risikofaktoren oder Schutzfaktoren bei CED dar.

Eine große Anzahl von Metaanalysen zu einzelnen Umweltfaktoren beschäftigt sich mit der Frage, welche Risikofaktoren für die Entstehung einer CED gesichert sind und welche Umweltfaktoren vor Entwicklung einer CED schützen. In einem sogenannten „Umbrella-Reviews“, eine Metaanalyse der Metaanalysen, führen Piovani et al. eine Risikoabschätzung zu 71 Umweltfaktoren, die mit Lebensstil und Hygiene, Medikation, chirurgischen Eingriffen, Ernährung, Mikroorganismen und Impfungen verbunden sind, durch. Folgende Faktoren wurden identifiziert:

\begin{tabular}{ll}
\hline Schutz vor der Entstehung einer CED & $\begin{array}{l}\text { Risikoerhöhung für die Entwicklung einer } \\
\text { CED }\end{array}$ \\
\hline Hohe körperliche Aktivität (MC) (-37\%) & Rauchen (MC) (+76\%) \\
\hline Stillen (CED) (-26\%) & Leben in der Stadt (CED) (+45\%) \\
\hline $\begin{array}{l}\text { Benutzung von Doppelbetten (MC) } \\
(-44 \%)\end{array}$ & Appendektomie (MC) (+61\%) \\
\hline Teekonsum (CU) (-31\%) & Tonsillektomie (MC) (+37\%) \\
\hline $\begin{array}{l}\text { Hohe Folsäure-Spiegel (CED) (-57\%) } \\
\text { Hohe Vitamin-D-Spiegel (MC) (-38\%) }\end{array}$ & Antibiotikaexposition (CED) (+57\%) \\
\hline \begin{tabular}{l} 
Onfektionen mit H. pylori (CED) (-57\%) \\
\hline $\begin{array}{l}\text { Frucht- und Gemüsektiva (CED) (+31\%) } \\
(-29 \%)\end{array}$
\end{tabular} & \begin{tabular}{l} 
Konsum von “soft drinks" (CU) (+69\%) \\
\hline Vitamin-D-Defizienz (CED) (+64\%)
\end{tabular} \\
\hline
\end{tabular}

Auch wenn die Effektstärken der einzelnen Faktoren für den klinischen Alltag nicht groß sind, da sie nur relativ geringe Risikoerhöhungen oder erniedrigungen darstellen eignen sich die Daten für die Beratung der Patienten zum Lebensstil. Möglicherweise ergibt sich aus der Kombination verschiedener Faktoren ein Schutz vor der Krankheitsmanifestation.

\section{Bodini G, Zanella C, Crespi M, Lo Pumo S, Demarzo MG, Savarino E2, Savarino V, Giannini EG. A Randomized, 6-wk Trial of a Low FODMAP Diet in Patients with Inflammatory Bowel Disease. Nutrition. 2019 Nov-Dec;67-68:110542.}

Eine FODMAP-arme Ernährung ist bei Patienten mit Reizdarmsyndrom ein weit verbreiteter Ansatz ion der Therapie. Das Akronym FODMAP steht für fermentierbare Oligo-, Di- und (and) Monosaccharide und Polyole, also häufig genannte „Unruhestifter" wie Fruktane, Lactose, Fruktose und Sorbit. Die Kohlenhydratverbindungen kommen in vielen Lebensmitteln vor, beispielswiese in Weizen, Roggen, Hülsenfrüchten, verschiedenen Obst- und Gemüsesorten, Milch, Joghurt und Honig Diesen Molekülen ist gemein, dass sie im Dünndarm nicht oder nur unvollständig resorbiert werden. Sie gelangen daher in das Kolon und werden dort von der Mikrobiota zu kurzkettigen Fettsäuren wie Acetat, Propionat und Butyrat abgebaut, wobei Gase, vor allem Wasserstoff, Kohlendioxid und Methan, entstehen. Die Gase können so Symptome verursachen. Zudem wirken die FODMAP osmotisch, sie erhöhen die Wassersekretion in das Darmlumen. Ein hoher Konsum von FODMAPs kann zu Verdauungsbeschwerden, wie Durchfall, Blähungen und Bauchschmerzen, führen.

In einer 6-wöchigen randomisierten Studie untersuchten Bodini et al. bei 55 CED-Patienten in Remission bzw. mit milder Krankheitsaktivität den Effekt einer Low-FODMAP-Diät im Vergleich zu einer Standarddiät. Als Zielkriterien diente der Harvey- 
Bradshaw-Index $(\mathrm{HBI})$ bei MC $(\mathrm{n}=35)$, der partielle MAYOScore bei CU $(n=20)$ sowie die fäkale Calprotectinkonzentration (fC) im Stuhl sowie der IBD-Q als Lebensqualitätsparameter im Vorher-nachher-Vergleich. Folgende Ergebnisse wurden erhoben:

\begin{tabular}{lcclll}
\hline & \multicolumn{2}{l}{ Low-FODMAP-Diät } & & \multicolumn{2}{l}{ Standard-Diät } \\
\cline { 2 - 3 } & Vorher & Nachher & & Vorher & Nachher \\
\hline HBI & 4 & 3 & & 3 & 3 \\
pMAYO & $2(2-3)$ & $1(1-3)$ & & $2(2-3)$ & $2(1-2)$ \\
fC (mg/kg Stuhl) & $76(50-286)$ & $50(51-81)^{*}$ & & $91(51-144)$ & $87(50-236)$ \\
IBD-Q & $166(139-182)$ & $177(155-188)^{*}$ & & $181(153-197)$ & $166(153-200)$ \\
\hline
\end{tabular}

Nach Ansicht der Autoren ist eine Low-FODMAP-Diät sicher und mit einer Verbesserung der fäkalen Entzündungsmarker und der Lebensqualität verbunden.

\section{Levine A, Wine E, Assa A, Sigall Boneh R, Shaoul R, Kori M, Cohen S, Peleg S, Shamaly H, On A, Millman P, Abramas L, Ziv-Baran T, Grant S, Abitbol G, Dunn KA, Bielawski JP, Van Limbergen J. Crohn's Disease Exclusion Diet Plus Partial Enteral Nutrition Induces Sustained Remission in a Randomized Controlled Trial. Gastroenterology. 2019 Aug;157(2):440-450.}

Eine alleinige enterale Diät (Formular Diät) zur Therapie des akuten Schubes bei Kindern und Jugendlichen ist ein etabliertes, von Leitlinien empfohlenes Behandlungskonzept. Problematisch ist die Adhärenz mit Verzicht auf alle weiteren anderen Nahrungsmittel über 6-8 Wochen. In der Pathogenese des M. Crohn wird einer „western style Diät“ mit Störung der gastrointestinalen Barriere und Veränderung des Mikrobioms eine wichtige Rolle zugeordnet. Vor diesem Hintergrund wurde für 6 Wochen die Kombination einer M.-Crohn-Exklusionsdiät mit partieller enteraler Ernährung $(\mathrm{n}=40)$ gegen die alleinige enterale Ernährung $(\mathrm{n}=34)$ bei Kindern und Jugendlichen (4-18 Jahre; mittleres Alter 14 Jahre) mit wenig- bis moderat-aktivem M. Crohn getestet. Für die Exklusionsdiät wurden Nahrungsmittel ausgewählt, die als Substrat für Proteobakterien dienen (Ballaststoffe, Stärke). Nahrungsmittel mit hohem tierischem Fettgehalt, Fetten aus anderen Nahrungsmitteln, Weizen, rotes und verarbeitetes Fleisch oder Nahrungsmittel mit hohem Gehalt von Süßstoffen, Carragenen und Sulfiten wurden aus der Diät ausgeschlossen. Um dieses erfolgreich umsetzen zu können, wurde für Patienten und Eltern eine Schulung initiiert, wöchentliche Telefonvisiten durchgeführt und eine telefonische Hotline sowie Informationsmaterial über das Internet angeboten. Der Anteil der Elementardiät durfte nicht mehr als $1250 \mathrm{ml} /$ Tag betragen. Folgende Lebensmittel waren erlaubt:

\begin{tabular}{|c|c|}
\hline Phase 1 (erste sechs Wochen) & Phase 2 (6.-12. Woche) \\
\hline $\begin{array}{l}\text { Obligatorische Lebensmittel } \\
\text { Hühnerbrust } 150-200 \mathrm{~g} / \mathrm{d} \\
\text { 2Eier pro Tag } \\
2 \text { Bananen/Tag } \\
1 \text { Apfel/Tag } \\
2 \text { Kartoffeln (müssen gekocht und wieder } \\
\text { gekühlt (über Nacht) werden) }\end{array}$ & $\begin{array}{l}\text { Obligatorische Lebensmittel } \\
\text { Hühnerbrust } 150-200 \mathrm{~g} / \mathrm{d} \\
2 \text { Eier pro Tag } \\
2 \text { Bananen/Tag } \\
1 \text { Apfel/Tag } \\
2 \text { Kartoffeln (müssen gekocht und wieder } \\
\text { gekühlt (über Nacht) werden) }\end{array}$ \\
\hline laubte Nahrungsmittel & Erlaubte Nahrungsmittel \\
\hline $\begin{array}{l}\text { Frische Erdbeeren } \\
\text { Melone (1 Scheibe) } \\
\text { Reismehl } \\
\text { Weißer Reis und Reisnudeln (unbegrenzt) } \\
2 \text { Tomaten (zum Kochen) } \\
2 \text { Gurken (mittlere Größe) } \\
2 \text { halbe Avocado } \\
1 \text { Karotte } \\
\text { Kopfsalat (3 Blätter) } \\
\text { Zwiebeln } \\
\text { Grüne Kräuter } \\
1 \text { Glas frisch gepresster Orangensaft } \\
\text { Wasser, Mineralwasser } \\
\text { Salz, Pfeffer, Paprika, Kurkuma, Gelbwurz } \\
3 \text { Esslöffel Honig } \\
4 \text { Teelöffel Zucker } \\
\text { Ingwer, Knoblauchzehen, Zitronen, } \\
\text { Limonen }\end{array}$ & $\begin{array}{l}\text { Frische Erdbeeren } \\
\text { Melone (1 Scheibe) } \\
\text { Reismehl } \\
\text { Weißer Reis und Reisnudeln (unbegrenzt) } \\
2 \text { Tomaten (zum Kochen) } \\
2 \text { Gurken (mittlere Größe) } \\
2 \text { halbe Avocado } \\
1 \text { Karotte } \\
\text { Kopfsalat (3 Blätter) } \\
\text { Zwiebeln } \\
\text { Grüne Kräuter } \\
1 \text { Glas frisch gepresster Orangensaft } \\
\text { Wasser, Mineralwasser } \\
\text { Salz, Pfeffer, Paprika, Kurkuma, Gelbwurz } \\
3 \text { Esslöffel Honig } \\
4 \text { Teelöffel Zucker } \\
\text { Ingwer, Knoblauchzehen, Zitronen, } \\
\text { Limonen } \\
\text { Eine Scheibe Brot pro Tag } \\
3 \text { TL Linsen oder Erbsen } \\
6 \text { Mandeln oder Walnusshälften } \\
\text { Quinoa }\end{array}$ \\
\hline
\end{tabular}

Folgende Ergebnisse wurden zu Woche 6 erhoben:

\begin{tabular}{lcc}
\hline Parameter & Exklusionsdiätgruppe & Elementardiät-gruppe \\
\hline Toleranz & $97,5 \%$ & $73,7 \%$ \\
Gute Compliance & $82,5 \%$ & $76,5 \%$ \\
Ansprechen & $85 \%$ & $85,3 \%$ \\
Remission & $80 \%$ & $73,5 \%$ \\
Fäkales Calprotectin & $3^{\prime} 126=>1^{\prime} 744 \mathrm{mg} / \mathrm{kg}$ & $2^{\prime} 647=1{ }^{\prime} 021 \mathrm{mg} / \mathrm{kg}$ \\
CRP & $23,6 \mathrm{mg} / \mathrm{L}=>5 \mathrm{mg} / \mathrm{L}$ & $24 \mathrm{mg} / \mathrm{L}=>4,1 \mathrm{mg} / \mathrm{L}$ \\
Anteil der Patienten mit normaler & & $56 \%=>56 \%$ \\
intestinaler Permeabilität & $46 \%=>69 \%$ & \\
\hline
\end{tabular}

$\mathrm{Zu}$ Woche 12 waren in der M.-Crohn-Exklusionsdiät mit Elementardiät-Gruppe $75,6 \%$ in einer steroidfreien Remission im Vergleich $\mathrm{zu} 45,1 \%$ in der alleinigen Elementardiätgruppe. Die Kombination aus Elementardiät und strenger Schonkost verbesserte die Akzeptanz der Therapie ohne den Behandlungseffekt zu beeinträchtigen.

\section{Godny L, Maharshak N, Reshef L, Goren I, Yahav L, Fliss-Isakov N, Gophna U, Tulchinsky H, Dotan I. Fruit Consumption is Associated with Alterations in Microbial Composition and Lower Rates of Pouchitis. J Crohns Colitis. 2019 Sep 27;13(10):1265-1272.}

Patienten mit ileoanalem Pouch bei Colitis ulcerosa haben ein erhöhtes Risiko für die Entwicklung einer Pouchitis. Die gute Wirksamkeit von Antibiotika bei einer Pouchitis spricht u.a. für die Bedeutung des Mikrobioms bei der Entstehung dieser Komplikation. Vor diesem Hintergrund untersuchten die Arbeitsgruppe um I. Dotan aus Israel den Einfluss der Diät auf das Mikrobiom und das Risiko an einer Pouchitis zu erkranken. Hierfür führten 172 Patienten ausführliche Ernährungstagebücher; bei 75 Patienten (22 Patienten mit Pouchitis, 53 Patienten ohne Pouchitis) wur- 
de eine Mikrobiomanalyse durchgeführt. In der gesamten Kohorte hatten bei Rekrutierung für die Studie 39 Patienten keine Pouchitis. Patienten, deren Fruchtkonsum unterhalb der niedrigsten Perzentile lag $(<1,45$ Einheiten pro Tag) hatten die höchste Gefahr an einer Pouchitis zu erkranken im Vergleich mit Patienten mit hohem/höchstem Konsum (30,8\% vs. 3,8\%, p = 0,03). Der Obstkonsum korrelierte mit der mikrobiellen Diversität und der Häufigkeit bestimmter Taxa wie Faecalibacteria-, Lachnospira-, Ruminococcaceae-Species. Eine Reduktion des Fruchtkonsums führte zu einem Anstieg des Pouchitisrisikos und Abnahme der Diversität $[\Delta=-0,8 \pm 0,3, p=0,008]$.

\section{Behrens G, Gredner T, Stock C, Leitzmann MF, Brenner H, Mons U. Cancers Due to Excess Weight, Low Physical Activity, and Unhealthy Diet. Dtsch Arztebl Int. 2018 Sep 3;115(35-36):578-585.}

Patienten mit Übergewicht, geringer körperlicher Aktivität, hohem Wurst-, Fleisch- und Salzkonsum sowie geringer Zufuhr von Ballaststoffen, Obst und Gemüse haben ein erhöhtes Krebsrisiko.

Auf der Basis von geschlechts- und altersgruppenspezifischen Bevölkerungsprojektionen sowie von Krebsinzidenz- und Expositionsdaten und publizierten Risikoschätzern haben die Forschenden Zahlen und Anteile (populationsattributable Fraktionen, PAF) für die 35- bis 84-jährige Bevölkerung in Deutschland für das Jahr 2018 geschätzt.

Eine beträchtliche Zahl an den für 2018 zu erwartenden Krebsneuerkrankungen ist auf Übergewicht, geringe körperliche Aktivität und ungesunde Ernährung zurückzuführen:

$\begin{array}{lc}\text { Übergewicht } & 30^{\prime} 567(7 \%) \\ \text { Geringe körperliche Aktivität } & 27^{\prime} 081(6 \%) \\ \text { Geringe Ballaststoffzufuhr } & 14^{\prime} 474(3 \%) \\ \text { Geringe Obst- und Gemüsezufuhr } & 447(2 \%) \\ \text { Wurstverzehr } & 99^{\prime} 454(2 \%) \\ \text { Hoher Verzehr von rotem Fleisch } & 1 \text { ''687 }(0,4 \%) \\ \text { Hoher Salzkonsum } & 1^{\prime} 204(0,3 \%)\end{array}$

Übergewicht ist ein Hauptrisikofaktor für Gebärmutter-, Nieren- und Leberkrebs (PAF $=24-35 \%)$. Geringe körperliche Aktivität trägt wesentlich zum Auftreten von Gebärmutter-, Nierenund Lungenkrebs (PAF $=15-19 \%)$ bei. Ernährungsfaktoren sind vor allem bei Darm-, Lungen- und Brustkrebs beteiligt (PAF = 9-16\%).
Hilfiker R, Meichtry A, Eicher M, Nilsson Balfe L, Knols RH, Verra ML, Taeymans J. Exercise and Other NonPharmaceutical Interventions for Cancer-Related Fatigue in Patients during or after Cancer Treatment: A Systematic Review Incorporating an IndirectComparisons Meta-Analysis. Br J Sports Med. 2018 May;52(10):651-658.

Ziel dieses systematischen Reviews mit Meta-Analyse war es herauszufinden, welchen Effekt unterschiedliche Typen von Sport und Bewegung und andere nichtpharmakologische Verfahren auf Erschöpfung im Zusammenhang mit Krebs und ihre Behandlung (cancer related fatigue - CRF) haben. Artikel aus PubMed, Cochrane CENTRAL und publizierten Meta-Analysen stellten die Quelle dar.

Randomisierte Studien wurden eingeschlossen, die bis 2017 publiziert wurden und eine Form von Bewegung, Sport oder andere nichtpharmakologische Intervention zur Reduktion von CRF bei jeder Form von Krebs während oder nach der Behandlung untersuchten.

Die Gefahr der Verzerrung (risk of bias) wurde mit den PEDro Kriterien geschätzt und eine Bayessche Netzwerk-Meta-Analyse mit Zufallseffekten wurde gerechnet.

245 Studien wurden eingeschlossen. Im Vergleich mit konventionellen Behandlungen während einer Krebsbehandlung waren Entspannungsübungen die wirksamsten Verfahren (standardisierte Mittelwertsdifferenz [SMD] d $=-0,77 ; 95 \%$ glaubwürdiges Intervall [CrI -1,22 bis -0,31]). Die anderen Verfahren hatten alle signifikante, moderate bis große Effektstärken: Massage $(d=-0,78$; $-1,55$ bis -0,01), kognitive Verhaltenstherapie mit körperlicher Aktivität $(\mathrm{d}=-0,72 ;-1,43$ bis $-0,09)$, aerobes Training kombiniert mit Widerstandstraining $(\mathrm{d}=-0,67 ;-1,01$ bis $-0,34)$, Widerstandstraining allein $(d=-0,53 ;-1,01$ bis $-0,03)$, aerobes Training $(d=-0,53$; $-0,80$ bis $-0,26)$ und Yoga $(\mathrm{d}=-0,51 ;-1,01$ bis 0,0$)$. Im Anschluss an die Krebsbehandlung war Yoga die Intervention mit dem größten Effekt $(d=-0,68 ;-0,93$ bis $-0,43)$. Die anderen Verfahren zeigten alle signifikante, mittlere bis kleine Effektgrößen: kombiniertes aerobes und Widerstandstraining ( $\mathrm{d}=-0,5 ;-0,66$ bis $-0,34)$, kombinierte kognitive Verhaltenstherapie $(\mathrm{d}=-0,45 ;-0,7$ bis $-0,21)$, Tai Chi $(\mathrm{d}=-0,45 ;-0,84$ bis $-0,06)$, kognitive VT $(\mathrm{d}=-0,42 ;-0,58$ bis $-0,25)$, Widerstandstraining $(\mathrm{d}=-0,35 ;-0,63$ bis $-0,08)$ und aerobes Training allein $(d=-0,33 ;-0,51$ bis $-0,16)$.

Patienten können also unter verschiedenen wirksamen Maßnahmen der Bewegungstherapie und anderen nichtpharmakologischen Interventionen wählen, um damit ihre CRF zu lindern. 